\title{
Crucial CD8+ T-lymphocyte cytotoxic role in amphotericin B nanospheres efficacy against experimental visceral leishmaniasis
}

Sofia A. Costa Lima ${ }^{a, \square, 1}$, Ricardo Silvestre ${ }^{a}$, Daniela Barros ${ }^{a}$, Joana Cunha $^{\mathrm{a}, \mathrm{b}}$, Maria Teresa Baltazar ${ }^{\mathrm{c}, \mathrm{d}, \mathrm{e}}$, Ricardo Jorge Dinis Oliveira ${ }^{\mathrm{c}, \mathrm{d}, \mathrm{e}, \mathrm{f}}$, Anabela Cordeiro-da-Silva ${ }^{\mathrm{a}, \mathrm{g}}$

${ }^{a}$ IBMC-INEB, Infection and Immunology Unit, Parasite Disease Group, University of Porto, Portugal;

b Instituto de Ciências Biomédicas Abel Salazar e Faculdade de Medicina, Universidade do Porto, Portugal;

${ }^{c}$ IINFACTS - Institute of Research and Advanced Training in Health Sciences and Technologies, Department of Sciences, Advanced Institute of Health Sciences-North (ISCS-N), CESPU, CRL, Gandra, Portugal;

d Department of Legal Medicine and Forensic Sciences, Faculty of Medicine, University of Porto, Porto, Portugal;

${ }^{e}$ REQUIMTE, Laboratory of Toxicology, Department of Biological Sciences, Faculty of Pharmacy, University of Porto, Porto, Portugal

${ }^{f}$ Forensic Sciences Center (CENCIFOR), Portugal;

${ }^{g}$ Department of Biological Sciences, Faculty of Pharmacy, University of Porto, Portugal.

E-mail address: slima@ff.up.pt (S.A. Costa Lima).

Keywords: Amphotericin B; Poly(D,L-lactide-co-glycolide) nanospheres; Visceral leishmaniasis; Immune-modulation; CD8 ${ }^{+} \mathrm{T}$ lymphocytes

Originally published at Nanomedicine: Nanotechnology, Biology and Medicine, 2014, 10(5):1021-30, doi: 10.1016/j.nano.2013.12.013, Elsevier 


\section{ABSTRACT}

This work aims to develop poly(d,I-lactide-co-glycolide) (PLGA)-nanospheres containing amphotericin $\mathrm{B}(\mathrm{AmB})$ with suitable physicochemical properties and anti-parasitic activity for visceral leishmaniasis (VL) therapy. When compared with unloaded-PLGA-nanospheres, the AmB-loaded PLGA-nanospheres displayed an increased particle size without affecting the polydispersity and its negative surface charge. AmB stability in the PLGA-nanospheres was $>90 \%$ over 60 -days at $30^{\circ} \mathrm{C}$. The AmB-PLGA-nanospheres demonstrated significant in vitro and in vivo efficacy and preferential accumulation in the visceral organs. In addition, an immune-modulatory effect was observed in mice treated with AmB-PLGA-nanospheres, correlating with improved treatment efficacy. The in vitro cytotoxic response of the $T$ lymphocytes revealed that AmB-PLGA-nanospheres efficacy against VL infection was strictly due to the action of $\mathrm{CD} 8^{+}-$but not $\mathrm{CD} 4^{+}-\mathrm{T}$ lymphocytes. Overall, we demonstrate a crucial role for $\mathrm{CD}^{+}$cytotoxic $\mathrm{T}$ lymphocytes in the efficacy of AmB-PLGA nanospheres, which could represent a potent and affordable alternative for VL therapy.

\section{FROM THE CLINICAL EDITOR}

This study demonstrates a crucial role for CD8+ T lymphocytes in eliminating visceral leishmaniasis in a murine model by enhancing the cytotoxic efficacy of CD8+ T-cells via amphotericin-B-PLGA nanospheres, paving a way to a unique, potentially more potent and cost-effective therapeutic strategy.

\section{INTRODUCTION}

Amphotericin B (AmB) is a polyene antibiotic, approved by the FDA, which is commonly used to treat invasive fungal infections and as second-line drug in the treatment of visceral leishmaniasis (VL). AmB is a produced by Streptomyces sp. and has low solubility at physiological $\mathrm{pH}(<0.1 \mathrm{mg} / \mathrm{mL})$, which restrains the development of pharmaceutical formulations for oral and parenteral administrations $(1,2)$. The connection between its physico-chemical properties, pharmacokinetics and pharmacodynamics remains uncertain (3-5). It has a high $\mathrm{VL}$ curative rate but requires prolonged period of treatment and hospitalization, leading to renal toxicity, among other severe adverse reactions. Indeed, the extensive association of AmB with low density lipoproteins (LDL) and the internalization of the AmB-LDL complex by kidney cells rich in high affinity LDL receptors responsible for the nephrotoxicity is well known $(4,6)$.

Leishmaniasis is a protozoan disease spread in nearly one hundred countries worldwide and is listed as the second major human neglected tropical disease in terms of mortality and morbidity with 350 million people living at risk, and a prevalence of 2 million cases a year, of which 0.5 million cases are of VL, a potentially fatal disease if left untreated. However, the treatment options are limited and unsatisfactory since most available drugs require parenteral 
administration and present serious toxicity with the occurrence of resistant strains contributing to hamper their success.

Drug delivery systems (DDSs) are a well-established approach to improve the therapeutic efficiency of drugs with concomitant reduction of drug toxicity. Several AmB lipid formulations (AmBisome ${ }^{\circledR}$, Amphocil ${ }^{\circledR}$ and Abelcet ${ }^{\circledR}$ ) and other DDSs have been developed in the past few years, but only the liposomal formulation AmBisome $\AA$ has become a standard treatment for $\operatorname{VL}(6,7)$. Recently, a single-course therapy of $10 \mathrm{mg} / \mathrm{kg}$ has been shown to cure $95 \%$ of $\mathrm{VL}$ patients in India (8). AmBisome ${ }^{\circledR}$ is significantly less toxic than the conventional AmB colloidal dispersion with sodium deoxycholate (Fungizone $\AA$ ) developed in $1958(9,10)$ since the former alters the pharmacokinetics, distribution and excretion profiles of AmB by decreasing free $A m B$ concentrations in plasma as a result of encapsulation of the drug in

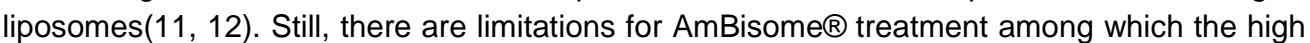
cost and temperature stability (recommended $25^{\circ} \mathrm{C}$ for unopened vials of lyophilized material and $2-8{ }^{\circ} \mathrm{C} / 24 \mathrm{~h}$ for reconstituted vials, accordingly to the manufacturer) comprise the major drawbacks. ${ }^{5}$ Moreover, AmB repeated administrations result in its accumulation due to slow elimination from the body, ultimately leading to nephrotoxicity (13). In view of all these facts, the search for a safer, more potent and especially more cost-effective AmB delivery system still remains. Recently, new DDSs for AmB have been described but some issues related with efficacy, toxicity and stability sill remain (14-19).

The present work aims to produce a nanospheres-based delivery system for AmB in an attempt to develop a cost-effective formulation with reduced toxicity, increased temperature stability and retaining its efficiency to VL treatment. To overcome AmB's drawbacks, we considered that a low-dose therapeutic system for AmB is desirable, aiming specifically to reduce the side-effects while maintaining its anti-parasitic efficacy. In order to develop such a delivery system for AmB, polymeric nanospheres (NS) based on Poly(D,L-lactide-co-glycolide) (PLGA) were selected due to their sustained-release characteristics, easy scale up, simple transformation, small particle size, biodegradability and biocompatibility properties (20). These are crucial properties to ensure an intracellular delivery and sustained release of drugs at a therapeutically relevant level (21). Here, we report the preparation and physicochemical characterization of the AmB-PLGA-NS followed by the in vitro and in vivo evaluation of the anti-leishmanial activity in comparison to free AmB or its commercially available lipid formulations. In parallel, we evaluated the in vivo AmB's distribution and drug-induced toxicity in several organs and unravel the probable interaction of this novel nano-formulation with the immune response.

\section{METHODS}

Detailed descriptions of the materials and methods can be found in the Supplementary Material

Ethics statement. All procedures involving animals were approved by and performed in accordance with the Portuguese National Authority for Animal Health license (0042/000/2012) and, the requirements and regulations laid down by the ethical review of IBMC-INEB Animal Ethics Committee. 
Preparation and physical characterization of AmB-loaded PLGA nanospheres. Drug loaded PLGA-NS were prepared based on the nanoprecipitation method (22). The mean particle size, size distribution and zeta potential ( $\zeta$-potential) of the nanoformulations were determined with a Zetasizer Nano ZS detector (Malvern Instruments, Worcestershire, UK). The morphology of NS was observed by transmission electronic microscopy (TEM) (TEM Jeol JEM-1400, Tokyo, Japan). AmB loading on the PLGA-NS was determined directly by measuring the amount of drug entrapped in the NS by an Ultra Performance Liquid Chromatography (UPLC) method described in the Supplementary Material (Table S1).

Parasites, cell culture, infection of mice and treatment schedules. For experimental infections, BALB/c mice (6-8 weeks old) were injected intraperitoneally (i.p.) with $10^{8}$ stationary phase promastigotes. Infected mice (6 mice/group) received i.v. injection of a single-shot treatment of drug-free PLGA-NS (1.5 mg/mouse), AmBisome ${ }^{\circledR}(1 \mathrm{mg} / \mathrm{kg})$, and a single or three consecutive daily doses of AmB-PLGA-NS (1 mg/kg AmB associated with $1.5 \mathrm{mg}$ of PLGA-NS) after 14-days of infection.

In vivo AmB distribution. The analysis of $A m B$ in tissues samples was based on a previously described analytical method (23). Homogenized tissues were used for the UPLC analysis and the limit of AmB quantification was $20 \mathrm{ng} / \mathrm{mL}$.

T cell proliferation assay. T cell proliferation assay was performed with splenocytes from different experimental groups of treated and untreated mice isolated by mechanical disruption. After $96 \mathrm{~h}$ the proliferation of splenic lymphocytes labeled with carboxyfluorescein succinimidyl ester was evaluated by flow cytometry, and T-cell populations identified with APC-conjugated anti-mouse CD4 and PB-conjugated anti-mouse CD8.

Analysis of cytokines and total nitrites production. Secreted cytokines were quantified in the splenocytes culture supernatants of differently treated infected mice by ELISA as recommended by the manufacturer (BioLegend, San Diego CA, USA). The total nitrite oxide (NO) content was quantified in supernatants of $72 \mathrm{~h}$-culture of splenocytes recovered from the different treatment animal groups stimulated, or not, with soluble Leishmania antigens (SLA) using the Griess method (24).

In vitro cell-mediated cytotoxicity assays. The in vitro cytotoxicity assay was accomplished using a LIVE/DEAD cell-mediated cytotoxicity kit (Molecular Probes, Invitrogen Life Technologies, Alfragide, Portugal) according to the manufacturer's protocol.

\section{RESULTS}

Physicochemical characterization of nanospheres: size, zeta potential, morphology, drug content, storage stability and in vitro release. AmB was encapsulated into PLGANS by nanoprecipitation, ${ }^{22}$ following an optimization step described in Supplementary Material (Table S2). The physicochemical properties of the optimized NS such as particle size, polydispersity (PDI), $\xi$-potential and drug encapsulation efficiency (EE) were studied and are summarized in Table 1.

The nanoformulations displayed mean diameter of $187.3 \pm 1.3$ to $221.5 \pm 6.6 \mathrm{~nm}$ for empty and AmB-PLGA-NS, respectively, with low PDI. Loading of the PLGA-NS with AmB led to a statistically significant increase on the size $(P<0.05)$ without disturbing the PDI or the 
surface charge as shown by constant $\zeta$-potential values around - $18 \mathrm{mV}$. TEM photographs revealed that empty (Figure $1, A$ ) and AmB-PLGA-NS (Figure 1, $B$ ) were found to be homogeneous and spherically shaped. Moreover, the AmB-PLGA-NS could be obtained in a reproducible and industrial-sized production within a short period of time $(6 \mathrm{~h})$. The encapsulation efficiency was determined by UPLC analysis of AmB-loaded nanoformulations. The AmB loading was calculated to be about $130 \mu \mathrm{g} / \mathrm{mg}$ of PLGA, which represented an EE of $65 \%$.

The influence of $\mathrm{pH}$ on the AmB release profile from the PLGA-NS for 10-days is shown on Figure 1, C. For both physiologic and acidic $\mathrm{pH}$ conditions the NS displayed a biphasic pattern characterized by a burst release of $\mathrm{AmB}$ that lasted approximately $6 \mathrm{~h}$, followed by a continuous release of the drug for at least 10-days. Yet, the effect observed during the first phase of release was much more drastic at acidic than at physiologic $\mathrm{pH}(P<0.05)$.

The AmB-PLGA and unloaded PLGA-NS did not show any signs of aggregation during the purification steps and remained stable upon storage at $4{ }^{\circ} \mathrm{C}$ for at least 60 -days, as no significant changes were observed on their particle size, PDI and $\zeta$-potential (Figure 1, D-E). With the increase of the storage period the nanoformulations were found slightly less negative than the freshly prepared nanoformulations $(P<0.05)$ (Figure $1, D)$ with higher tendency to aggregate $(\mathrm{PDI}>0.1)$ and a non-significant size increase (Figure $1, E$ ). No modification was found on the AmB content when the PLGA-NS were stored at $4{ }^{\circ} \mathrm{C}$ up to 60 -days (data not shown).

To evaluate the in vitro stability of AmB-PLGA-NS under sub-tropical and tropical temperatures, the nanoformulations were incubated at 30 and $42{ }^{\circ} \mathrm{C}$. Regarding the drug content, the temperature did not affect the stability of AmB in PLGA-NS, exceeding $94.38 \% \pm 3.56 \%$ (Figure S1D, light grey) and $86.12 \% \pm 4.28 \%$ (Figure S1D, dark grey) of its initial concentration after 30 -days at $30^{\circ} \mathrm{C}$ or $42^{\circ} \mathrm{C}$, respectively. Nevertheless, this decrease on the AmB content inside the PLGA-NS did not affect its efficacy. Indeed, the antileishmanial activity of AmB-PLGA-NS stored at $30{ }^{\circ} \mathrm{C}$ for 60 -days exhibited an $\mathrm{IC}_{50}$ value of $0.16 \pm 0.03 \mu \mathrm{g} / \mathrm{mL}$ against intracellular $L$. infantum amastigotes in THP1 differentiated macrophages, which is remarkably similar to the one observed with freshly prepared NS $(0.13 \pm 0.02 \mu \mathrm{g} / \mathrm{mL}, \mathrm{p}>0.05)$.

In vivo efficacy of the AmB-PLGA nanospheres. AmB-PLGA-NS led to a marked in vitro anti-leishmanial outcome (Figure S2, Table S3) hence the efficacy of the AmB-PLGA-NS was assessed in a VL susceptible murine model by a single- or a three-daily dose treatment at $1 \mathrm{mg} / \mathrm{kg}$. In all treated groups a significant reduction $(P<0.001)$ of the parasite load was found on the splenic and hepatic tissues (Figure 2, $A$ and $B$ ). When given in a single-dose or 3 -daily doses at $1 \mathrm{mg} / \mathrm{kg}$, AmB-PLGA-NS promoted a reduction of $98.6 \% \pm 1.4 \%$ and $99.3 \% \pm 0.5 \%$, respectively, in the splenic parasites corresponding to approximately 100 -fold decrease, as compared to the vehicle control. Concerning the liver, a less effective outcome was observed, with $90.6 \% \pm 0.7 \%$ and $98.7 \% \pm 0.8 \%$ parasite reduction for AmB-PLGA-NS given in single or 3-daily doses, respectively, related to the vehicle control. Importantly, the AmB-PLGA-NS led to a more pronounced reduction on the splenic parasites when compared with $\mathrm{AmBisome} \AA$ irrespective of the number of doses given $(P<0.05)$ (Figure $2, A)$. In the liver, a 3-consecutive daily dose was as effective as AmBisome ${ }^{\circledR}$ in reducing the parasite burden (Figure 2, $B$ ).

In vivo AmB distribution. In order to clarify the high splenic efficacy of AmB-PLGA-NS we were interested in understanding the distribution pattern of $A m B$ when given as Fungizone ${ }^{\circledR}$ 
$(0.8 \mathrm{mg} / \mathrm{kg})$, AmBisome ${ }^{\circledR}(1 \mathrm{mg} / \mathrm{kg})$ and PLGA NS $(1 \mathrm{mg} / \mathrm{kg})$ by i.v. to infected animals. Fungizone ${ }^{\circledR}$ was chosen for comparison as another amphotericin $B$ formulation clinically used. The results of tissue distribution of $A m B 2 \mathrm{~h}$ following i.v. administration of the three formulations are summarized in Table 2.

Following i.v. administration of PLGA-NS and AmBisome $\AA$, AmB accumulates mainly in the spleen and liver, in comparison to other tissues (kidney, lung and heart). Furthermore, an impressive amount of AmB from the PLGA-NS is focused on the spleen, being approximately 2 -fold less present in the liver. In opposition, AmB delivered by AmBisome ${ }^{\circledR}$ accumulates twice more in the liver than in the spleen. Even so, the overall concentrations in these two Leishmania-target organs are not significantly different for those two AmB formulations.

Toxicological studies. The safety of a new VL treatment is as fundamental as its efficacy. By assessing the oxidative stress it is possible to ascertain tissue toxicity elicited by the administration of the AmB treatments (25). Therefore, we evaluated biomarkers of oxidative stress in the liver and spleen, namely reduced (GSH) and oxidized glutathione (GSSG).

The measurement of the ratio of reduced GSH to GSSG in the liver showed a statistical significant decrease $(P<0.01)$ for PLGA-NS-, single- and 3-consecutive doses-AmB-PLGANS, and AmBisome®-treated groups when compared with the untreated group (Figure $3, A$ ). Similarly, in the spleen (Figure 3, B) a statistical decrease $(P<0.05)$ for PLGA-AmB-treated group was observed. The extent of drug-induced tissue toxicity by ROS was further determined by means of lipid peroxidation and myeloperoxidase activities in the liver and spleen, but no statistically significant differences were observed within all the experimental groups in the tissues analyzed (Figure S3).

AmB-PLGA nanospheres positively interact with the immune system. Further experiments were performed to evaluate if AmB-PLGA-NS induce a protective modulation of adaptive immunity besides the anti-leishmanial activity in VL mice model.

Splenocytes from AmB-PLGA-NS treated animals showed enhanced CD4 ${ }^{+}$and $\mathrm{CD} 8^{+}-\mathrm{T}$ cell proliferation in response to SLA $(P<0.001)$, while empty NS and AmBisome ${ }^{\circledR}$ treatment had little or no effect on lymphocytes proliferation (Figure 4, A). Even though the development of $\mathrm{T}$ cell specific proliferation was observed with a single-dose of AmB-PLGA-NS, the effect was more pronounced with the 3-consecutive daily administrations (Figure 4, $A$ ). The development of a T-cell specific response did not result from a quantitative modification of the spleen cellular populations (Figure S4) or to significant changes on their activation status (Figure S5). Instead, we detected a significant increase on the number of effector memory $\mathrm{CD}^{+}-\mathrm{T}$ cells (Figure $4, B$ ), and $\mathrm{CD}^{+}{ }^{-}$-T cells to a lower extent ( 4\%, Figure S6), after treatment with AmBPLGA-NS. Indeed, the analysis of the $\mathrm{CD}^{+}-\mathrm{T}$ cell phenotype obtained from AmB-PLGA-NS treated mice revealed a predominant effector memory subset $\left(C D 44^{+} C D 62 L^{\text {low }}\right)$ as compared to $\mathrm{CD}^{+}-\mathrm{T}$ cells from controls (Figure $4, B$ ). These qualitative changes in the lymphocytes subpopulations led us to investigate their function in terms of cytokine profile developed during AmB-PLGA-NS treatment. We detected a 1.5- $(P<0.05)$ and 2 -fold $(P<0.001)$ increase in the frequencies of $\mathrm{IFNY}^{+} \mathrm{CD}^{+}-\mathrm{T}$ cells and $2.6 \quad(P<0.01)$ and 5.1 -fold $(P<0.001)$ enhancement of IFN- $\mathrm{y}^{+}-\mathrm{CD} 8^{+} \mathrm{T}$ cells exclusively for the groups that received a single-dose and a 3-consecutive daily doses of AmB-PLGA-NS, respectively (Figure 4, C). Although AmBisome® has proved to elicit similar reductions on parasite burden, we did not detect any significant production of IFN-y in both splenic CD4 ${ }^{+}$or $C D 8^{+}-\mathrm{T}$ cells. In all the treated groups (PLGA, AmB-PLGA-NS and AmBisome®), IL-10 ${ }^{+}$producing $\mathrm{CD}^{+}$and $\mathrm{CD}^{+}-\mathrm{T}$ cell 
populations did not change with respect to untreated controls (Figure S7A, B). Moreover, the IFN- $\mathrm{y}^{+}$producing $\mathrm{CD}^{+}-\mathrm{T}$ cells developed in AmB-PLGA-NS treated mice failed to produce IL10 (data not shown). The cytokine quantification of splenocytes supernatants re-stimulated with SLA confirmed these findings. AmB-PLGA-NS treated mice exhibited IL-12 and IFN-y levels significantly higher $(P<0.001)$ in comparison with untreated, AmBisome ${ }^{\circledR}$ or empty PLGA-NS treated infected animals (Figure 4, D). Indeed, IL-12 was 2- and 3-fold higher in single and 3-consecutive daily doses, respectively, which may account for the increase of IFN$Y$ levels in 8 and 11.5-fold in the animals that received single and 3-doses, respectively, of AmB-PLGA-NS. In opposition, IL-10 secretion was found to be restrained in both AmB-PLGANS or AmBisome ${ }^{\circledR}$ treated infected animals (Figure 4,D). This cytokinic microenvironment ultimately resulted in an increase of NO production (Figure 4, E). The AmB-PLGA-NS treated animals had a 5- and 6-fold increase in nitrite production, for single and 3-consecutive daily doses, respectively in comparison with the infected untreated animals, while AmBisome® treated animals showed a 2 -fold increase when compared with infected untreated animals (Figure 4, E).

Leishmania-specific cell-mediated response by antigen-specific cytotoxic $\mathrm{T}$ lymphocytes or innate cells is a part of the effective immune response developed against the parasite (26). Antigen-specific cytotoxic T lymphocytes or innate cells are responsible for this cytotoxic activity. In this study, our results provided evidence that the immune-stimulatory effect of AmB-PLGA-NS treatment acts by creating an immunological environment unfavorable for parasite survival and growth. Thus, we have investigated if the AmB-PLGA-NS treatment is also able to induce a Leishmania-specific cytotoxic response. Splenocytes from all experimental treatment groups were stimulated ex vivo with SLA for 7-days, to favor the expansion of Leishmania-specific clones. The cytotoxic response of these cells was then evaluated against $L$. infantum-infected macrophages, revealing a dose-dependent lysis of the target cells (infected macrophages) (Figure 4, F). Indeed, splenocytes recovered from AmBPLGA-NS treated mice were able to elicit a statistically significant $(P<0.001)$ dose dependent lysis of the target cells of $31.5 \%$ and $28.2 \%$ for a 3 -consecutive daily doses and a single-dose, respectively, at 40:1 (effector: target) ratio. In opposition, empty PLGA-NS or AmBisome® treated infected mice showed no specific cytolysis (Figure 4, $F$ ). These results demonstrate that AmB-PLGA-NS induced the generation of Leishmania-specific effector cytotoxic $T$ cells that act synergistically with the anti-leishmanial activity of $A m B$ in the elimination of infected macrophages. Importantly, this AmB-PLGA-NS formulation induced a strong adaptive immune response in addition to the anti-parasitic effect, which is absent upon treatment with the AmBisome® formulation.

Efficacy of AmB-PLGA nanospheres is associated with $\mathrm{CD}^{+} \mathrm{T}$ cells. Our results demonstrate that the AmB-PLGA-NS amplified the cellular response to Leishmania infection manifested by the development of antigen-specific $T$ lymphocytes with the concomitant secretion of IFN- $\gamma$. Previous studies have demonstrated that $C D 4^{+}$and $C D 8^{+}-T$ cells play a crucial role in the control of Leishmania infection characterized by IFN-y secretion $(27,28)$. To ascertain the relevant cellular mechanisms responsible for the improved protection induced by the AmB-PLGA-NS, we separately assessed the contributive role of each player (IFN- $\gamma$ and $\mathrm{CD}^{+}-$, CD8 ${ }^{+}-\mathrm{T}$ cells). Surprisingly, AmB-PLGA-NS was as effective in reducing the parasite load in spleen and liver of IFN- $-1-1-$ mice as the wild type control (Figure S8). Since our results demonstrate a Leishmania-specific T cell response upon AmB-PLGA-NS treatment (Figure 4, $F$ ), we investigated separately the potential role of $\mathrm{CD} 4^{+}$- and $\mathrm{CD} 8^{+}-\mathrm{T}$ cells. To do so, we have depleted each population before AmB-PLGA-NS treatment by injecting L. infantum infected $\mathrm{BALB} / \mathrm{c}$ mice with anti-CD8 or anti-CD4 monoclonal antibody (Figure S9). Remarkably, the treatment with AmB-PLGA-NS failed to control the infection in $\mathrm{CD}^{+}$depleted mice (Figure 5, 
A). Mice depleted of $\mathrm{CD} 4^{+}$cells but maintaining functional $\mathrm{CD} 8^{+}-\mathrm{T}$ cells were fully capable to elicit a reduction on the parasite burden after AmB-PLGA-NS treatment (Figure 5, A). In addition, the depletion of $\mathrm{CD}^{+}-\mathrm{T}$ cells abrogated the cytotoxic activity of AmB-PLGA-NSpulsed splenocytes against infected host macrophage cells (Figure $5, B$ ) while the depletion of $\mathrm{CD}^{+}-\mathrm{T}$ cells had little effect. Finally, only $\mathrm{CD} 8^{+}-\mathrm{T}$ cells purified from the spleen of AmB-PLGANS treated infected mice exhibited similarly a cytotoxic activity upon infected host macrophages (Figure 5, C). These data demonstrate the crucial role for Leishmania-specific cytotoxic $C D 8^{+}-T$ effector cells in the killing of $L$. infantum-infected macrophages in mice that have previously received AmB-PLGA-NS.

\section{RESULTS}

A low cost polymeric system for AmB using PLGA nanospheres was described. Our approach produced a tropically stable and safe DDS with an insight on the splenic cellular immune mechanisms responsible for the efficient response after VL treatment.

When defining a DDS for AmB it is important to consider the hydrophobicity of the compound and the importance of its controlled and sustained release into the surrounding environment. Biodegradable nanoparticles can be produced from natural or synthetic polymers, the latter being advantageous for prolonged release of the encapsulated therapeutic agents that can span for some days to several weeks (29). The polylactides and PLGA are among the most extensively investigated for drug delivery (30). As polyesters in nature, these polymers undergo hydrolysis in the body, forming moieties that are eventually removed from the body by the citric acid cycle. Introduced by Fessi (22) nanoprecipitation is a simple and mild method for the preparation of nanoparticles without the use of any preliminary emulsification. This method presents several advantages, since it does not involve aggressive steps (such as sonication, high temperatures and extended stirring rates), surfactants are not always needed and the organic solvents normally used do not display significant toxicity and, it is highly recommended for hydrophobic drugs $(31,32)$ Taking all these into consideration, the development of the AmB DDS was primarily based on the PLGA polymer and the nanoprecipitation method. Starting with a small AmB amount the drug was successfully loaded on PLGA-NS with a $65 \%$ EE value, which can be related to the nanospheres preparation method but also to the possible electrostatic interaction between capped carboxyl groups of PLGA and the positively charged primary amine group of AmB contributing to such a drug entrapment. Comparable drug EE values above $50 \%$ for AmB in PLGA nanoparticles are described in literature with higher initial drug loading values $(10 \%$ to $30 \%)(15,16)$. The AmB entrapment on the PLGA-NS leads to a statistically significant increase of about $30-\mathrm{nm}$ on the nanospheres size $(P<0.05)$ without affecting the morphology, PDI and $\zeta$-potential. It is well established that the stabilizer PVA forms a layer protecting the nanospheres from aggregation and that its residual amount remaining onto the nanospheres is relatively high due to strong attachment to the surface (33).

Assessment of the $\mathrm{AmB}$ in vitro release revealed $\mathrm{pH}$ dependence and since in $\mathrm{VL}$ the pathogen resides inside an acidic phagolysosome of macrophages, the AmB in vitro release profile suggests retention of the drug inside the NS on the mammalian cell cytoplasm and preferential release at the endosomal-lysosomal acidic pH. Indeed, after $6 \mathrm{~h}$ at $\mathrm{pH} 5.5$, $49.99 \% \pm 2.92 \%$ was found to be released from the NS whereas only $18.56 \% \pm 2.16 \%$ of AmB was released from the PLGA-NS under pH 7.4. Afterwards, a similar tendency of slower drug release was maintained for the period of 10 -days, revealing $88.40 \% \pm 3.56 \%$ and 
$60.23 \% \pm 2.03 \%$ AmB released from the PLGA-NS under acidic $\mathrm{pH}$ and physiological conditions, respectively.

Based on the accelerated temperature studies set out by the WHO to mimic tropical climate Zone 3 and 4 conditions (34), we analyzed the storage stability of the developed AmB-PLGA$\mathrm{NS}$ at $30{ }^{\circ} \mathrm{C}$ and $42^{\circ} \mathrm{C}$ in defined time points over a 60 -day period. Our nanoformulations improved AmB stability when compared with four AmB lipid formulations recently presented with remarkable temperature stability with about $80 \%$ after 30 to 60 days at $30{ }^{\circ} \mathrm{C}$ and $75 \%$ at $43^{\circ} \mathrm{C}$ in drug content (34). Besides, AmB-PLGA-NS stored for 60 -days at $30^{\circ} \mathrm{C}$ behave similarly to fresh preparations as observed by the determination of the in vitro activity on $L$. infantum intracellular amastigotes. All these data allow us to affirm that the AmB-PLGA-NS are a sub-tropically stable formulation.

The effectiveness of the AmB-PLGA-NS was assessed in a VL susceptible murine model. The

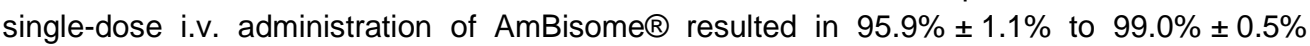
inhibition of spleen and liver parasites, respectively, consistent with literature reports $(35,36)$. When given in a single dose or 3-daily doses AmB-PLGA-NS led to a statistically significant reduction of splenic and liver parasites, corresponding to approximately $2-\log _{10}$ decrease, as compared to the vehicle control. Our data clearly demonstrate the advantageous efficacy of a single-dose of the AmB-PLGA-NS in reducing splenic parasites when compared to AmBisome ${ }^{\circledR}(P<0.05)$. This new DDS intravenously administered can be a promising approach for a structured VL treatment, preventing uncontrolled drug self-administration (e.g. oral route) as well as the emergence of AmB resistant strains.

In view of our in vivo results on VL murine model, we were interested in understanding the bio-distribution pattern of $A m B$ when given as Fungizone $\AA$, AmBisome $\AA$ and PLGA-NS by i.v. to infected animals. Both PLGA-NS $(\sim 200 \mathrm{~nm})$ and AmBisome ${ }^{\circledR}(\sim 100 \mathrm{~nm})$ are particulate systems recognized by the mononuclear phagocyte system (MPS) (primarily liver, spleen and, to a lesser extent, lungs), while Fungizone $\AA$, a colloidal micellar system with about $1 \mu \mathrm{m}$ size, is able to avoid the effect of the MPS (37). In fact, Fungizone® displayed a more homogenous distribution on the tissues, with an evident retention in the kidney, which may be correlated with its well-known nephrotoxicity $(38,39)$. Interestingly, the concentration of AmB in tissues $2 \mathrm{~h}$ following i.v. administration of PLGA-NS and AmBisome ${ }^{\circledR}$ reveals some similarities. In both cases, most of the drug is found in major MPS-riched tissues, liver and spleen. Given the bio-distribution of PLGA-NS and AmBisome ${ }^{\circledR}$ among the splenic and hepatic tissues, these findings support the higher effectiveness of PLGA-NS observed in the splenic parasites reduction in the VL murine model.

The safety of the VL treatment is as fundamental as its effectiveness. By assessing the oxidative stress, a marker of drug-induced organ toxicity (40), it is possible to ascertain tissue toxicity elicited by the administration of the AmB treatments (25). Here we have evaluated the effects of all the VL treatments on the liver and spleen by quantifying several oxidative stress indicators. Given that the developed AmB-PLGA-NS exhibited similar oxidative stress levels when compared to AmBisome $\AA$ and the untreated group it can be considered that identical drug-induced toxicity levels were caused in the liver or spleen. Nevertheless, extensive in vivo studies consisting of the assessment of nephrotoxicity and hepatotoxicity respectively of the new AmB-PLGA-NS will be addressed in the near future.

The profound impairment of the immune system of the infected host in VL is a major cause for incomplete efficacy of the anti-leishmanial chemotherapy, as the treatment success depends 
on the combined effect of the drug itself and the immune status of the host $(41,42)$. Our results suggest that AmB-PLGA-NS improve the inherent immune-modulatory efficacy of $\mathrm{AmB}$, most probably by directing towards a Th1 immune response, which is essential for a successful VL treatment. Th1 cells secreting large amounts of IFN- $\gamma$ will enhance cellular immunity by cytotoxic $T$ lymphocytes and macrophages (43). It has been described the requirement of continuous presence of IL-12 for sustaining Th1 immunity, providing protective cell-mediated immune response towards murine $L$. major infection (44). Indeed, our data show a substantial reduction in splenic parasite burden and high levels of IL-12 and IFN- $\mathrm{y}$, which verify these findings. Similarly, a high level of NO production in the AmB-PLGA-NS treated mice suggests the induction of NO-mediated macrophage effector mechanisms towards the control of parasite proliferation $(45,46)$. Moreover, IL-10 is considered to be the predominant immunosuppressive cytokine leading to susceptibility $(47,48)$. We detect a downregulation of IL-10 production either by lymphocytes or from other cellular origin. In this study, our results emphasize the importance of effective immune stimulation combined with anti-leishmanial activity for VL treatment. In the same way, it was described by others that AmB entrapped in stearylamine cationic liposomes was more effective in killing $L$. donovani and in retaining the immunomodulatory effect of free $\mathrm{AmB}$ on $\mathrm{CD} 4^{+}$and $\mathrm{CD} 8^{+}-\mathrm{T}$ cells for IFN-y production than AmBisome ${ }^{\circledR}$ (48). The AmB-PLGA-NS was able to preserve and develop the immunomodulatory effect already described for AmB (49), while AmBisome ${ }^{\circledR}$ did not, possibly due to different uptake mechanism and intracellular tracking of these two DDSs which may lead to different outcomes in the macrophage modulation and consequently $T$ cells function.

As a final point the efficacy of AmB-PLGA-NS was correlated to the CD8 ${ }^{+}-T$ cells function. Currently, it is known that $C D 8^{+}-T$ cells play an important role in the mechanisms for the cure and the resistance to Leishmania infection, either by the production of IFN- $\gamma$ and activation of macrophages, or by the direct killing of parasitized macrophages, or a combination of both effects $(27,28,49)$. Therefore, targeting CD8 ${ }^{+}-T$ cells responses may have great therapeutic potential against VL.

Here we have demonstrated that the incorporation of AmB in PLGA-NS is able to reduce experimental VL when $C D 8^{+}-\mathrm{T}$ cells are present. Although nanoparticles are considered an efficient tool for inducing potent immune responses and several vaccine applications have been described (50), to the best of our knowledge, we describe here for the first time the crucial role of $\mathrm{CD}^{+}-\mathrm{T}$ cells in the in vivo efficacy of a nano-therapy approach against intracellular Leishmania protozoan. Based on the present results, the developed AmB-PLGANS can be a promising approach for VL treatment, especially in tropical and subtropical developing countries where an affordable and effective approach is urgently needed.

\section{FUNDING}

This work was supported by Fundo Europeu de Desenvolvimento Regional (FEDER) funds through the Operational Competitiveness Program-COMPETE and by national funds through Fundação para a Ciência e a Tecnologia under projects FCOMP-01-0124-FEDER015718 (PTDC/SAU-ENB/113151/2009) and Fundação Caloust Gulbenkian under project P105348/2009. D.B. was supported by FCOMP-01-0124-FEDER-015718 (PTDC/SAUENB/113151/2009) project. S.A.C.L, J.C. T.B. and R.D-O. were supported by SFRH/BPD/37880/2007, SFRH/BD/48626/2008, SFRH/BD/65387/2009 and IF/01147/2013, respectively. R.S. was supported by Programa Ciência, financed by Programa Operacional 
Potencial Humano-Quadro de Referência Estratégica Nacional-Tipologia 4.2-Promoção do Emprego Científico, co-funded by Fundo Social Europeu and national funding from the Ministry of Science, Technology and Higher Education.

\section{COMPETING INTERESTS}

There are no competing interests to report.

\section{REFERENCES}

1. J. Torrado, R. Espada, M. Ballesteros, S. Torrado-Santiago Amphotericin B formulations and drug targeting J Pharm Sci, 7 (2008), pp. 2405-2425.

2. A. Lemke, A. Kiderlen, O. Kayser Amphotericin B Appl Microbiol Biotechnol, 68 (2005), pp. 151-162.

3. S. Sundar, H. Mehta, A. Suresh, S. Singh, M. Rai, H. Murray AmB treatment for Indian visceral leishmaniasis: conventional versus lipid formulations Clin Infect Dis, 38 (2004), pp. 377-383.

4. J. Brajtburg, J. Bolard Carrier effects on biological activity of AmB Clin Microbiol Rev, 9 (1996), pp. 512-531.

5. F. Meheus, M. Balasegaram, P. Olliaro Cost-effectiveness analysis of combination therapies for visceral leishmaniasis in the Indian subcontinent PLoS Negl Trop Dis, 4 (2010), p. 818.

6. L. Guo Amphotericin B, colloidal dispersion: an improved antifungal therapy Adv Drug Deliv Rev, 47 (2001), pp. 149-163.

7. J. Adler-Moore, R. Proffitt Amphotericin B lipid preparations: what are the differences Clin Microbiol Infect, 14 (2008), pp. 25-36.

8. S. Sundar, J. Chakravarty, D. Agarwal, M. Rai, H. Murray Single-dose liposomal amphotericin B for visceral leishmaniasis in India N Engl J Med, 362 (2010), pp. 504-512.

9. G. Boswell, D. Buell, I. Bekersky AmBisome (liposomal amphotericin B): a comparative review J Clin Pharmacol, 38 (1998), pp. 538-592.

10. T. Walsh, R. Finberg, C. Arndt, J. Hiemenz, C. Schwartz, D. Bodensteiner, et al. Liposomal amphotericin B for empirical therapy in patients with persistent fever and neutropenia N Engl J Med, 340 (1999), pp. 764-771.

11. I. Bekersky, R. Fielding, D. Buell, I. Lawrence Lipid-based amphotericin B formulations: from animals to man Pharm Sci Technol Today, 2 (1999), pp. 230-236.

12. I. Bekersky, R. Fielding, D. Dressler, J. Lee, D. Buell, T. Walsh Pharmacokinetics, excretion, and mass balanceof liposomal amphotericin B (AmBisome) and 
amphotericin B deoxycholate in humans Antimicrob Agents Chemother, 46 (2002), pp. 828-834.

13. A.J. Atkinson, J. Bennett Amphotericin B pharmacokinetics in humans Antimicrob Agents Chemother, 13 (1978), pp. 271-276.

14. S. Jung, D. Lim, S. Jung, J. Lee, K.-S. Jeong, H. Seong, et al. Amphotericin Bentrapping lipid nanoparticles and their in vitro and in vivo characteristics Eur $\mathrm{J}$ Pharm Sci, 37 (2009), pp. 313-320.

15. H. Van de Ven, C. Paulussen, P. Feijens, A. Matheeussen, P. Rombaut, P. Kayaert, et al. PLGA nanoparticles and nanosuspensions with amphotericin B: potent in vitro and in vivo alternatives to Fungizone and Am Bisome J Control Release, 161 (2012), pp. 795803.

16. J. Italia, A. Sharp, K. Carter, P. Warn, M. Kumar Peroral amphotericin B polymer nanoparticles lead to comparable or superior in vivo antifungal activity to that of intravenous Ambisome ${ }^{\circledR}$ or Fungizone ${ }^{\mathrm{TM}}$ PLoS One, 6 (2011), p. e25744.

17. Z. Yang, Y. Tan, M. Chen, L. Dian, Z. Shan, X. Peng, et al. Development of amphotericin B-loaded cubosomes through the solemuls technology for enhancing the oral bioavailability AAPS PharmSciTech, 13 (2012), pp. 1483-1491.

18. J. Jain, M. Jatana, A. Chakrabarti, N. Kumar Amphotericin-B-loaded polymersomes formulation (PAMBO) based on (PEG)3-PLA copolymers: an in vivo evaluation in a murine model Mol Pharm, 8 (2011), pp. 204-212.

19. V. Prajapati, K. Awasthi, S. Gautam, T. Yadav, M. Rai, O. Srivastava, et al. Targeted killing of Leishmania donovani in vivo and in vitro with amphotericin B attached to functionalized carbon nanotubes J Antimicrob Chemother, 66 (2011), pp. 874-879.

20. S. Vyas, S. Gupta Optimizing efficacy of amphotericin B through nanomodification Int J Nanomedicine, 1 (2006), pp. 417-432.

21. M. Chavanpatil, A. Khdair, J. Panyam Nanoparticles for cellular drug delivery: mechanisms and factors influencing delivery J Nanosci Nanotechnol, 6 (2006), pp. 26512663.

22. H. Fessi, F. Puisieux, J. Devissaguet, N. Ammoury, S. Benita Nanocapsule formation by interfacial polymer deposition following solvent displacement Int J Pharm, 55 (1989), pp. R1-R4.

23. R. Espada, J. Josa, S. Valdespina, M. Dea, M. Ballesteros, J. Alunda, et al. HPLC assay for determination of amphotericin B in biological samples Biomed Chromatogr, 22 (2008), pp. $402-407$.

24. A. Ding, C. Nathan, D. Stuehr Release of reactive nitrogen intermediates and reactive oxygen intermediates from mouse peritoneal macrophages. Comparison of activating cytokines and evidence for independent production J Immunol, 141 (1988), pp. 24072412. 
25. N. Paniker, S. Srivastava, E. Beutler Glutathione metabolism of the red cells. Effect of glutathione reductase deficiency on the stimulation of hexose monophosphate shunt under oxidative stress Biochim Biophys Acta, 215 (1970), pp. 456-460.

26. T. Bousoffara, H. Louzir, A. Ben Salah, K. Dellagi Analysis of granzyme B activity as a surrogate marker of Leishmania-specific cell-mediated cytotoxicity in zoonotic cutaneous leishmaniasis J Infect Dis, 189 (2004), pp. 1265-1273.

27. Z. Wang, S. Reiner, S. Zheng, D. Dalton, R. Locksley CD4 effector cells default to the Th2 pathway in interferon gamma-deficient mice infected with Leishmania major J Exp Med, 179 (1994), pp. 1367-1371.

28. Y. Belkaid, E. Von Stebut, S. Mendez, R. Lira, E. Caler, S. Bertholet, et al. CD8 ${ }^{+} \mathbf{T}$ cells are required for primary immunity in $\mathrm{C} 57 \mathrm{BL} / 6$ mice following low-dose, intradermal challenge with Leishmania major J Immunol, 168 (2002), pp. 3992-4000.

29. S.M. Moghimi, A.C. Hunter, J.C. Murray Long-circulating and target specific nanoparticles: theory to practice Pharmacol Rev, 53 (2001), pp. 283-318.

30. R.A. Jain The manufacturing techniques of various drug loaded biodegradable poly(lactide-co-glycolide) devices Biomaterials, 21 (2000), pp. 2475-2490.

31. J. Barichello, M. Morishita, K. Takayama, T. Nagai Encapsulation of hydrophilic and lipophilic drugs in PLGA nanoparticles by the nanoprecipitation method Drug Dev Ind Pharm, 25 (1999), pp. 471-476.

32. S. Vrignaud, J. Benoit, P. Saulnier Strategies for the nanoencapsulation of hydrophilic molecules in polymer-based nanoparticles Biomaterials, 33 (2011), pp. 8593-8604.

33. D. Quintanar-Guerrero, A. Ganem-Quintanar, E. Allémann, H. Fessi, E. Doelker Influence of the stabilizer coating layer on the purification and freeze-drying of poly(d, I-lactic acid) nanoparticles prepared by an emulsion-diffusion technique J Microencapsul, 15 (1998), pp. 107-119.

34. E. Wasan, P. Gershkovich, J. Zhao, X. Zhu, K. Werbovetz, R. Tidwell, et al. A novel tropically stable oral amphotericin B formulation (iCo-010) exhibits efficacy against visceral leishmaniasis in a murine model PLoS Negl Trop Dis, 4 (2010), p. e913.

35. V.K. Prajapati, K. Awasthi, T.P. Yadav, M. Rai, O.N. Srivastava, S. Sundar An oral formulation of amphotericin B attached to functionalized carbon nanotubes is an effective treatment for experimental visceral leishmaniasis $J$ Infect Dis, 205 (2012), pp. 333-336.

36. J. Gangneux, A. Sulahian, Y. Garin, R. Farinotti, F. Derouin Therapy of visceral leishmaniasis due to Leishmania infantum: experimental assessment of efficacy of Am Bisome Antimicrob Agents Chemother, 40 (1996), pp. 1214-1218.

37. H. Fukui, T. Koike, T. Nakagawa, A. Saheki, S. Sonoke, Y. Tomii, et al. Comparison of LNS-AmB, a novel low-dose formulation of amphotericin B with lipid nano-sphere (LNS), with commercial lipid-based formulations Int J Pharm, 267 (2003), pp. 101-112. 
38. R. Fielding, A. Singer, L. Wang, S. Babbar, L. Guo Relationship of pharmacokinetics and drug distribution in tissue to increased safety of amphotericin B colloidal dispersion in dogs Antimicrob Agents Chemother, 36 (1992), pp. 299-307.

39. I. Bekersky, G. Boswell, R. Hiles, R. Fielding, D. Buell, T. Walsh Safety, toxicokinetics and tissue distribution of long-term intravenous liposomal amphotericin B (AmBisome): a 91-day study in rats Pharm Res, 17 (2000), pp. 1494-1502.

40. C.V. Pereira, S. Nadanaciva, P.J. Oliveira, Y. Will The contribution of oxidative stress to drug-induced organ toxicity and its detection in vitro and in vivo Expert Opin Drug Metab Toxicol, 8 (2012), pp. 219-237.

41. P. Sharma, N. Singh, R. Garg, W. Haq, A. Dube Efficacy of human a-casein fragment (54-59) and its synthetic analogue compound 89/215 against Leishmania donovani in hamsters Peptides, 25 (2004), pp. 1873-1881.

42. E. Carvalho, R. Teixeira, W.J. Johnson Cell-mediated immunity in American visceral leishmaniasis: reversible immunosuppression during acute infection Infect Immun, 33 (1981), pp. 498-500.

43. S. Reiner, R. Locksley The regulation of immunity to Leishmania major Annu Rev Immunol, 13 (1995), pp. 151-177.

44. T. Joshi, S. Rodriguez, V. Perovic, I. Cockburn Stager pp. B7-H1 blockade increases survival of dysfunctional CD8(+) T cells and confers protection against Leishmania donovani infections PLoS Pathog, 5 (2009), p. e1000431.

45. R. Polley, S. Stager, S. Prickett, A. Maroof, S. Zubairi, D. Smith, et al. Adoptive immunotherapy against experimental visceral leishmaniasis with CD8 + T cells requires the presence of cognate antigen Infect Immun, 74 (2006), pp. 773-776.

46. J. Moon, B. Huang, D. Irvine Engineering nano- and microparticles to tune immunity Adv Mater, 24 (2012), pp. 3724-3746.

47. B.M. Owens, L. Beattie, J.W. Moore, N. Brown, J.L. Mann IL-10-producing Th1 cells and disease progression are regulated by distinct $\operatorname{CD11c(+)}$ cell populations during visceral leishmaniasis PLoS Pathog, 8 (2012), p. e1002827.

48. A. Banerjee, M. De, N. Ali Complete cure of experimental visceral leishmaniasis with amphotericin B in stearylamine-bearing cationic liposomes involves down-regulation of IL-10 and favorable T cell responses $\mathrm{J}$ Immunol, 181 (2008), pp. 1386-1398.

49. H. Murray, E. Brooks, J. DeVecchio, F. Heinzel Immunoenhancement combined with amphotericin B as treatment for experimental visceral leishmaniasis Antimicrob Agents Chemother, 47 (2003), pp. 2513-2517.

50. P. Tsagozis, E. Karagouni, E. Dotsika CD8(+) T cells with parasite-specific cytotoxic activity and aTc1 profile o fcytokine and chemokine secretion develop in experimental visceral leishmaniasis Parasite Immunol, 25 (2003), pp. 569-579. 


\section{TABLES \& FIGURES}

Table 1. Physicochemical characterization of the nanospheres

\begin{tabular}{lllll}
\hline Nanospheres & Size $(\mathrm{nm})$ & PDI & $\begin{array}{l}\xi \text {-potential } \\
(\mathrm{mV})\end{array}$ & $\begin{array}{l}\text { Encapsulation } \\
\text { efficiency }(\%)\end{array}$ \\
\hline PLGA-NS & $187.3 \pm 1.3$ & $0.06 \pm 0.02$ & $-18.2 \pm 0.8$ & NA \\
AmB-PLGA-NS & $221.5 \pm 6.6^{*}$ & $0.09 \pm 0.03$ & $-18.9 \pm 0.9$ & $65.4 \pm 4.7$ \\
\hline
\end{tabular}

Data are expressed as the mean \pm SEM $(n=6)$. NA, not applicable;

${ }^{*} P<0.05$, statistically significant when compared to empty nanospheres.

Table 2. Tissue distribution of AmB $2 \mathrm{~h}$ following i.v. administration of PLGA-NS, Fungizone $\AA$ and AmBisome®.

\begin{tabular}{|c|c|c|c|}
\hline \multirow[t]{2}{*}{ Tissue } & \multicolumn{3}{|c|}{ Treatment group } \\
\hline & Fungizone ${ }^{\otimes}$ & AmBisome & AmB-PLGA-NS \\
\hline Liver & $541 \pm 65$ & $5148 \pm 426$ & $2456 \pm 288$ \\
\hline Kidney & $895 \pm 81$ & $539 \pm 56$ & $184 \pm 26$ \\
\hline Spleen & $286 \pm 27$ & $2934 \pm 286$ & $4225 \pm 354$ \\
\hline Lung & $45 \pm 8$ & $215 \pm 18$ & $95 \pm 12$ \\
\hline Heart & $32 \pm 4$ & $248 \pm 22$ & $42 \pm 3$ \\
\hline
\end{tabular}

$\mathrm{ng} / \mathrm{g}$, mean $\pm \mathrm{SEM}, \mathrm{n}=6$. 


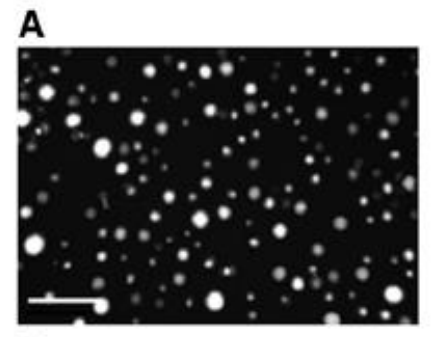

B
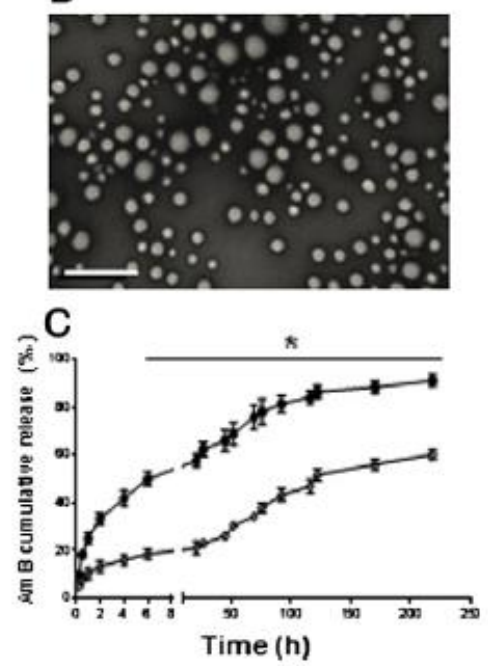

D
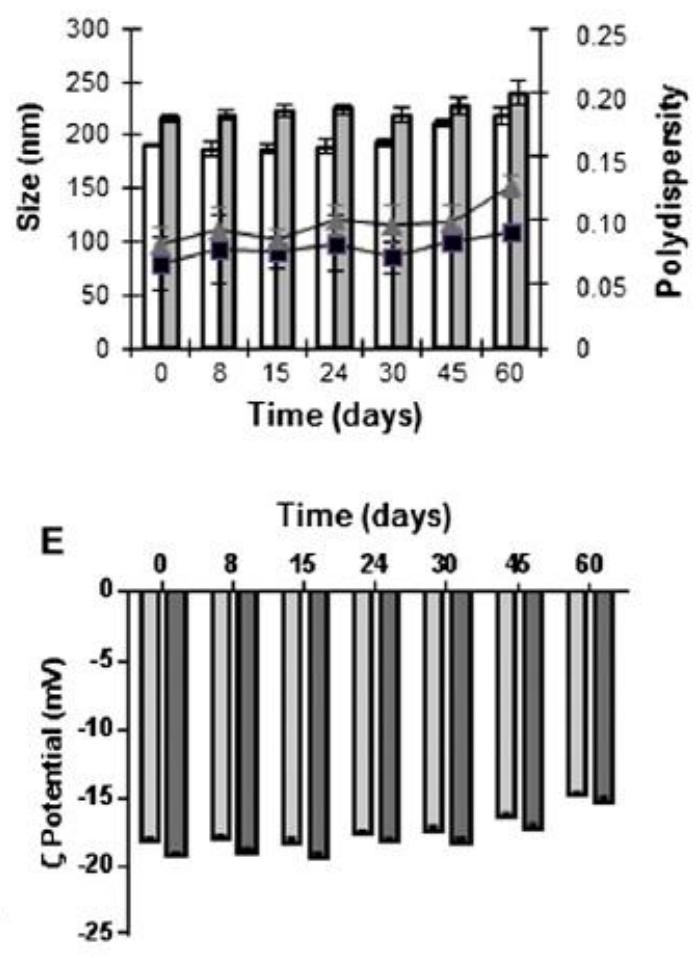

Figure 1. Characterization of the AmB-PLGA nanospheres. Morphology of unloaded (A) and AmB-loaded (B) PLGANS; scale bar is $500 \mathrm{~nm}$. (C) AmB in vitro release kinetics from PLGA-NS at pH $5.5(\bullet)$ and pH $7.4(\diamond)$; means \pm SDs $(n=5)$. (D) Storage stability for unloaded (white bars; squares) and AmB-loaded (dark bars; triangles) PLGA-NS at $4{ }^{\circ} \mathrm{C}$ for 2 -months according to size (bars) and PDI (lines) and (E) $\zeta$-potential. 

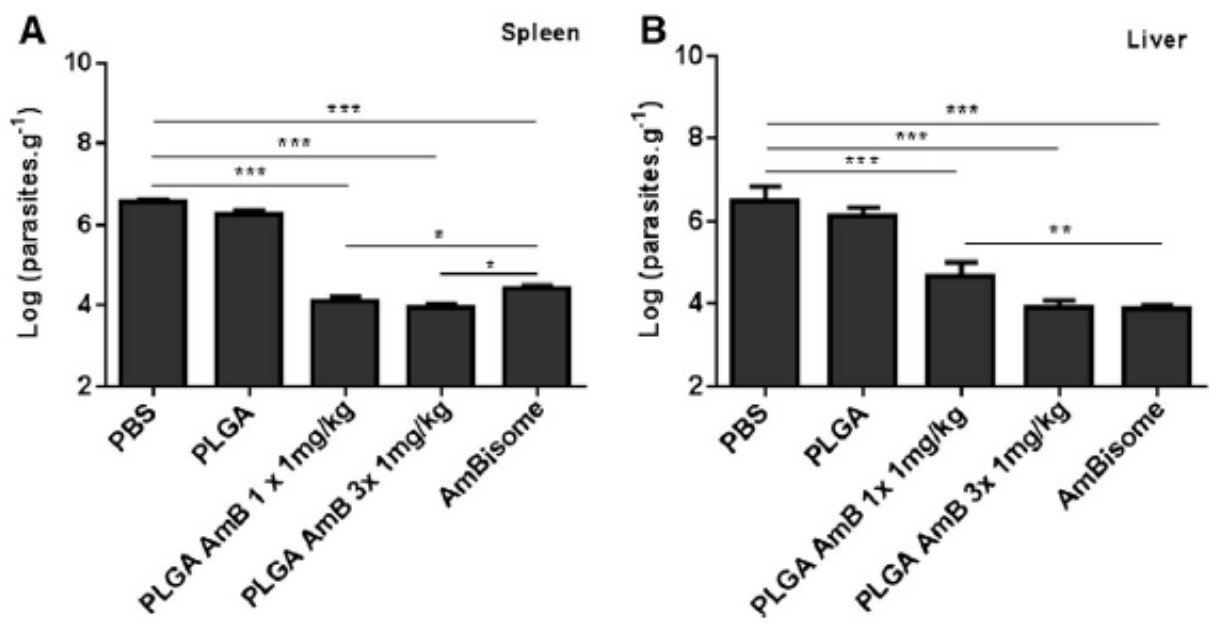

Figure 2. In vivo efficacy of AmB-PLGA nanospheres in L. infantum-infected mice. 3-days after the last administration, parasite burden was evaluated by limiting dilution assay in the spleen (A) and in the liver (B). Data represent the mean $\pm S D$ of a representative experiment from two carried out independently, with six mice per experimental group in each experiment ${ }^{\star} P<0.05,{ }^{\star *} P<0.01$ and ${ }^{* * *} P<0.001$ in comparison with untreated group. 

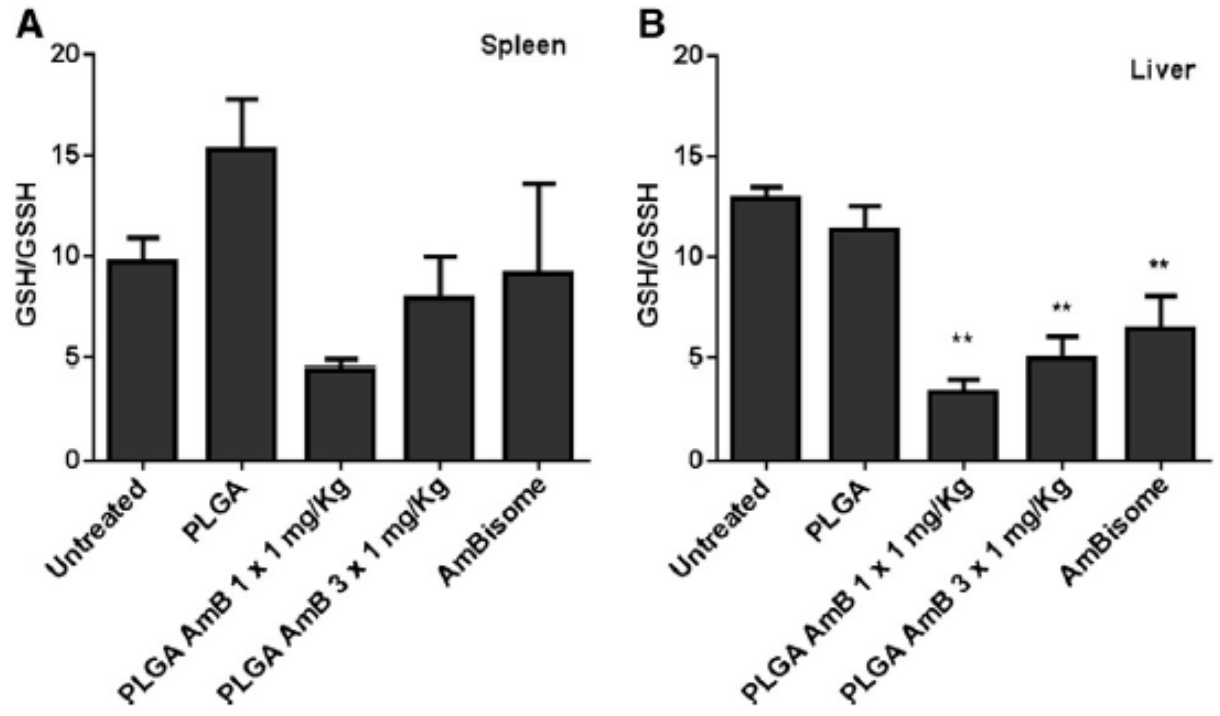

Figure 3. Measurement of the ratio of reduced glutathione to oxidized glutathione. (A) Liver and (B) spleen of Leishmania-infected mice untreated or treated. Values are given as mean \pm SEM $(n=4)$. The comparison between untreated infected group and the different treatment groups was made with the one-way analysis of variance (ANOVA) with Dunnett's multiple comparison test. 
A

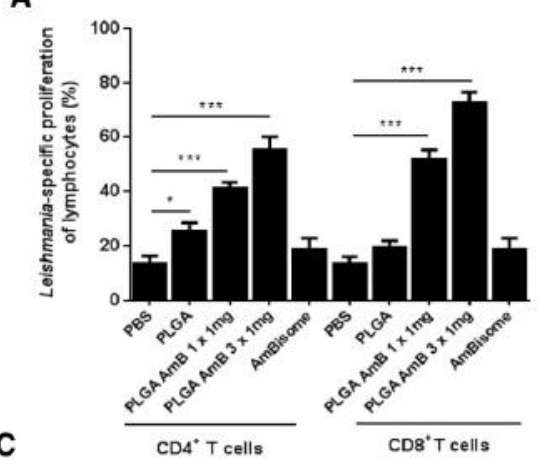

B
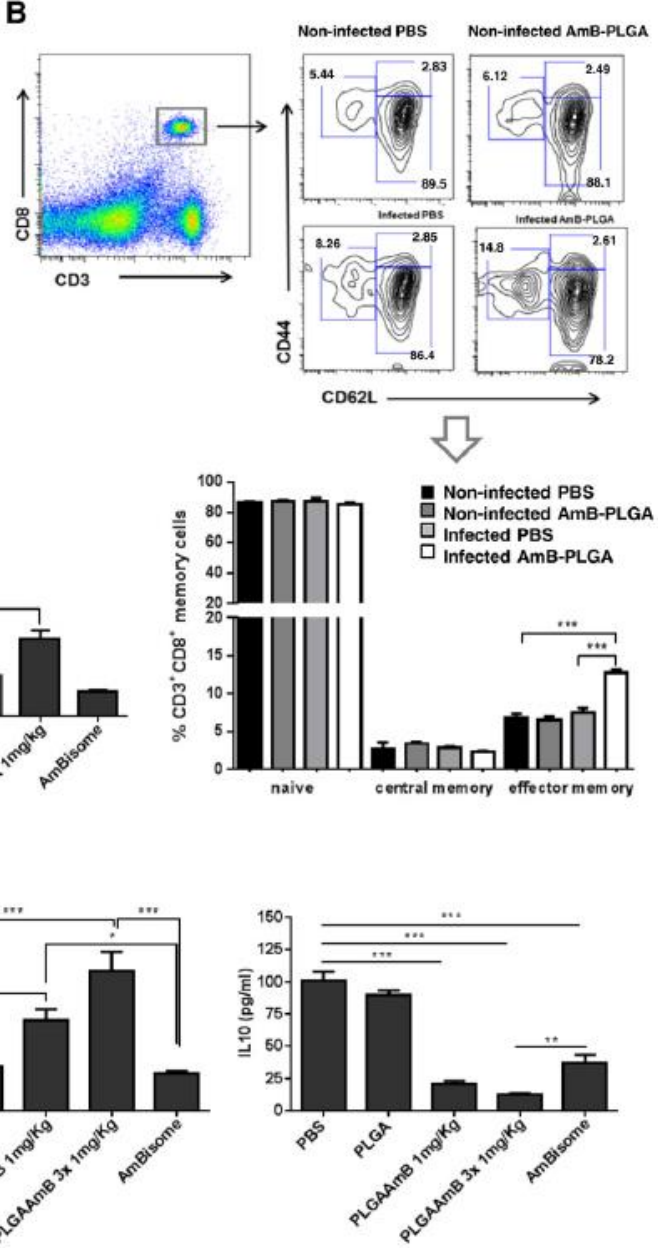

E
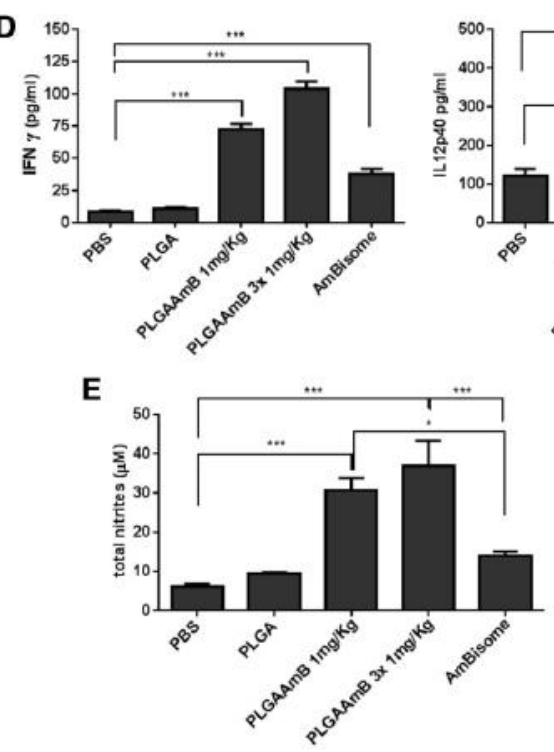

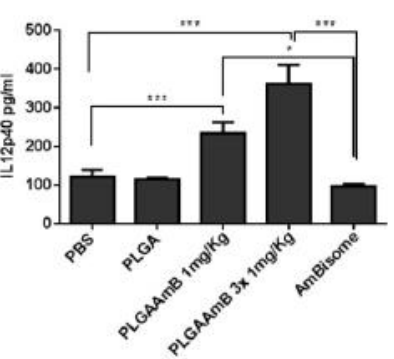

$\mathbf{F}$
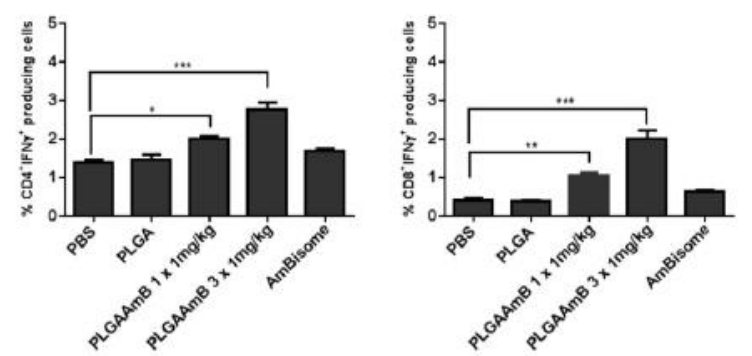

Figure 4. In vivo mechanism of AmB-PLGA nanospheres in L. infantum-infected mice. (A)/n vitro proliferation of splenic cells of differently treated mice. (B) Phenotype of CD8 ${ }^{+}$memory T cells. (C) Intracellular and secreted IFN- $\gamma$, $\mathrm{IL}-12 \mathrm{p} 40$ and IL-10 production of total $\mathrm{CD} 4^{+}-$and $\mathrm{CD} 8^{+}-\mathrm{T}$ cells from all experimental groups. (E) NO production. (F) AmB-PLGA-NS treatment causes a Leishmania-specific cytotoxic response. Data represent the mean \pm SD of a representative experiment from two carried out independently, with six mice per experimental group in each experiment ${ }^{\star} P<0.05$, ${ }^{* *} P<0.01$ and ${ }^{* \star *} P<0.001$ in comparison with untreated group. 


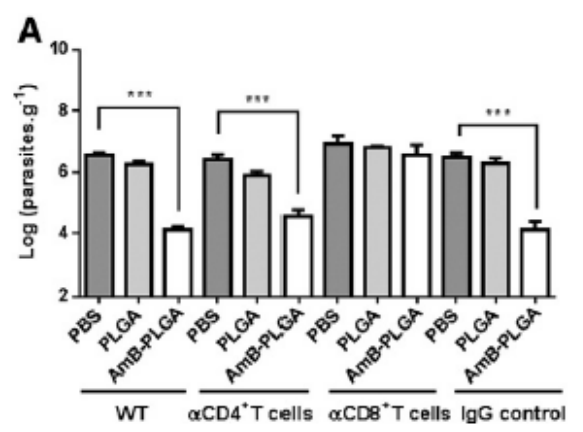

B

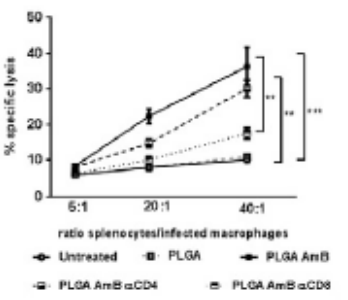

C

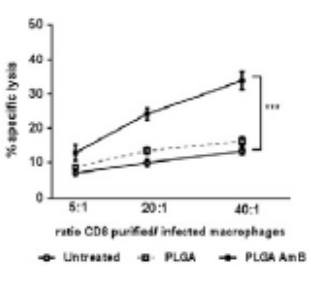

Figure 5. CD8 $8^{+}-T$ cells are crucial for the in vivo efficacy of AmB-PLGA nanospheres in L. infantum-infected mice. (A) Parasite load in PBS, PLGA-NS or AmB-PLGA-NS treated mice depleted of $\mathrm{CD} 4^{+}$and $\mathrm{CD} 8^{+}-\mathrm{T}$ cells prior treatment. Untreated and (PBS) treated mice with IgG were used as controls. (B, C) AmB-PLGA-NS treatment causes a Leishmania-specific cytotoxic response dependent on $C D 8^{+}-T$ cells. Data represent the mean $\pm S D$ of a representative experiment from two carried out independently, with four mice per experimental group in each experiment ${ }^{*} \mathrm{P}<0.05,{ }^{* *} \mathrm{P}<0.01$ and ${ }^{* * *} \mathrm{P}<0.001$ in comparison with untreated group. 


\section{GRAPHICAL ABSTRACT}

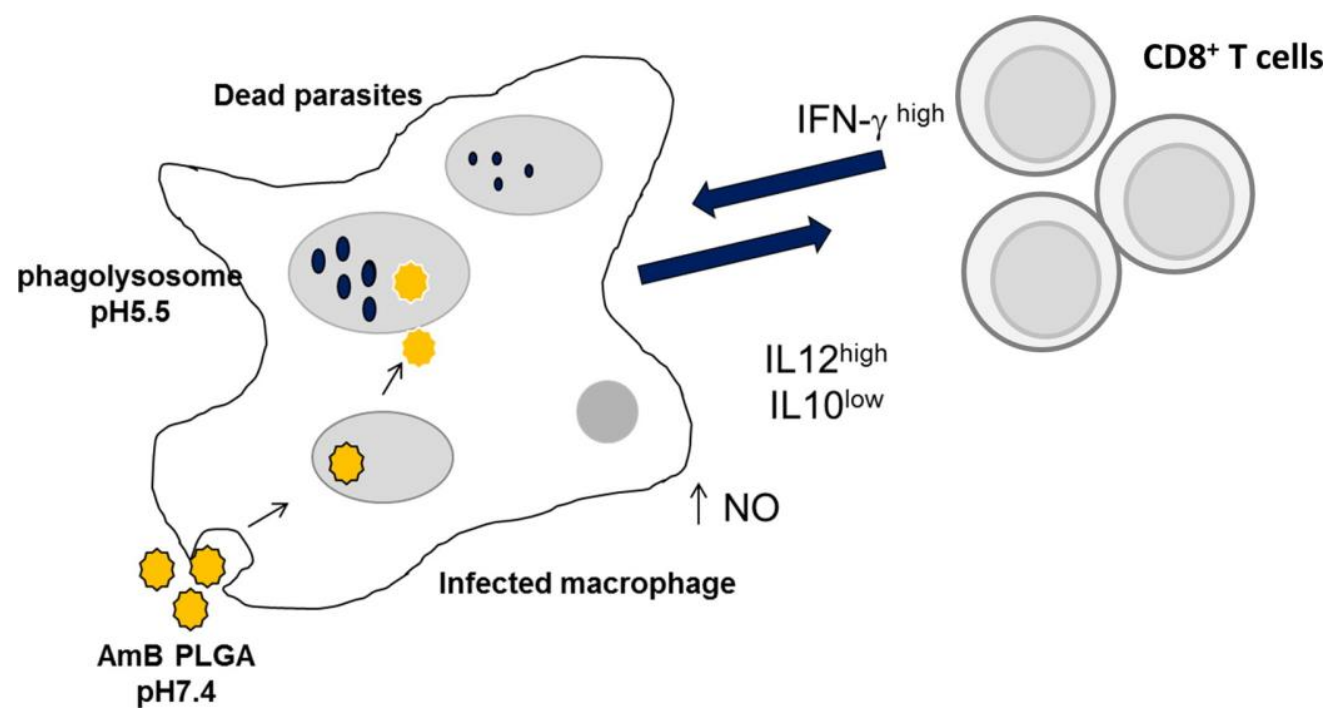

Proposed mechanism of action of AmB-PLGA nanospheres on Leishmania-infected macrophages and interaction with $\mathrm{CD} 8^{+} \mathrm{T}$ cells. 